\title{
Correction to: Decreasing thickness and remodeling of ligamentum flavum after oblique lumbar interbody fusion
}

\author{
Akaworn Mahatthanatrakul ${ }^{1} \cdot$ Hyeun Sung Kim ${ }^{2} \cdot$ Guang-Xun Lin $^{3} \cdot$ Jin-Sung Kim ${ }^{4}$
}

Published online: 7 July 2020

(C) Springer-Verlag GmbH Germany, part of Springer Nature 2020

Correction to: Neuroradiology (2020)

https://doi.org/10.1007/s00234-020-02414-y

In the article "Decreasing Thickness and Remodeling of Ligamentum Flavum After Oblique Lumbar Interbody Fusion", Fig. 2 captions contained mistakes as descriptions for the Figure sections were not assigned correctly.

The correct Fig. 2 captions are:

Fig. 2 Magnetic resonance imaging measurement. a Spinal canal cross-sectional area. b Ligamentum flavum thickness calculated by the average of left and right side thickness of ligamentum flavum at the thickest part. $\mathbf{c}$ Ligamentum flavum area. d Spinal canal width. e Foraminal area

The online version of the original article can be found at https:/doi.org/ 10.1007/s00234-020-02414-y

Jin-Sung Kim

mdlukekim@gmail.com

1 Department of Orthopaedics, Naresuan University Hospital, Phitsanulok, Thailand

2 Department of Neurosurgery, Nanoori Gangnam Hospital, Seoul, South Korea

3 Department of Orthopedics, The First Affiliated Hospital of Xiamen University, Xiamen, People's Republic of China

4 Spine Center, Department of Neurosurgery, Seoul St. Mary's Hospital, College of Medicine, The Catholic University of Korea, Seoul, South Korea 